\title{
Tuberin-heterozygous cell line TSC2ang1 as a model for tuberous sclerosis-associated skin lesions
}

\author{
PAWEL K. WLODARSKI ${ }^{1}$, RADOSLAW MAKSYM ${ }^{1}$, MONIKA OLDAK ${ }^{1}$, \\ SERGIUSZ JOZWIAK $^{2}$, ANDRZEJ WOJCIK ${ }^{3,4}$ and JAROSLAW JOZWIAK ${ }^{1}$ \\ ${ }^{1}$ Department of Histology and Embryology, Center for Biostructure Research, Medical University of Warsaw; \\ ${ }^{2}$ Department of Pediatric Neurology, The Children's Memorial Health Institute; ${ }^{3}$ Department of Radiation \\ Biology and Health Protection, Institute of Nuclear Chemistry and Technology, Warsaw; ${ }^{4}$ Department \\ of Radiobiology and Immunology, Swietokrzyska Academy, Institute of Biology, Kielce, Poland
}

Received August 2, 2007; Accepted September 20, 2007

\begin{abstract}
Tuberous sclerosis (TS), neurological disorder manifesting with the formation of tumors in numerous organ systems, is a disease associated with the upregulation of mammalian target of rapamycin (mTOR) pathway. It has been found that in healthy individuals two tumor suppressor genes, TSC1 and TSC2, encoding proteins called hamartin and tuberin, respectively, are responsible for the control over mTOR kinase. Loss of one of these genes constitutes the genetic background of TS. In the current study, we aimed at evaluating the fitness of the only TS-associated sarcoma cell line deposited in American Tissue Culture Collection, TSC2ang1, for the in vitro studies on TS. We found that the line shows a stable chromosome pattern with typical Robertsonian translocations. Similarly to primary tumors from TS patients, TSC2ang1 cells respond to rapamycininduced mTOR inhibition. The cells demonstrate activation of both Akt and Erk pathways, but inhibition of neither of them is as effective as mTOR suppression when considering proliferation potential. Based on these results we propose TSC2ang1 as a good and stable model for pathophysiological and pharmacological studies on skin lesions in TS.
\end{abstract}

\section{Introduction}

Tuberous sclerosis (TS), an autosomal dominant genetic disease, is caused by mutation of one of two tumor suppressor proteins: hamartin (TSC1) or tuberin (TSC2) $(1,2)$. TSC1 and TSC2 form an intercellular protein complex with GTPaseactivating properties towards a small $G$ protein Rheb (Ras

Correspondence to: Dr Jaroslaw Jozwiak, Department of Histology and Embryology, Center for Biostructure Research, Medical University of Warsaw, ul. Chalubinskiego 5, 02-004 Warsaw, Poland

E-mail: jaroslaw.jozwiak@gmail.com

Key words: TSC2ang1, cell line, tuberous sclerosis, skin lesions protein enriched in brain). It has been established that TSC2 can be phosphorylated by two kinases, Akt/PKB (protein kinase B) and Erk (extracellular signal-regulated protein kinase), and that these phosphorylations are deactivating $(3,4)$, leading to suppression of TSC1/TSC2 complex activity towards Rheb (reviewed in refs. 5,6). In turn, Rheb in its active form activates mTOR (mammalian target of rapamycin). mTOR is responsible for control of translation of a large portion of intracellular proteins, including those participating in the promotion of cell cycle, such as cyclin D1 or ornithine decarboxylase $(7,8)$. Thus, active mTOR is often associated with cell cycle progression and oncogenic transformation of cells (9).

Inactivating second-hit mutations in TSC1 or TSC2 have been found in numerous types of TS-associated tumors (10-13), but most authors agree that such mutations cannot be found in the brain (14-16). Thus, current research concentrates on answering the question: how can mTOR be hyperactive in the presence of wild-type hamartin and tuberin. One of the potential mechanisms relates to their post-translational inactivation by protein kinases such as Akt/PKB or Erk. There are several reports on hyperactivation of $\mathrm{Akt} / \mathrm{PKB}$ or Erk in human TS samples $(3,17,18)$.

TS is a natural model for evaluating the influence of mTOR on cellular processes. However, scant availability of biopsy material from TS patients encourages to look for other sources of cells with disturbed mTOR-signaling. So far there is no good in vitro model of TS. Pharmacological research is performed in knock-out mice or Eker rats $\left(\mathrm{TSC} 2^{+/-}\right)$, while in vitro assays, because of the lack of established cell lines from patients, utilize animal lines, however, no consensus as to which is the most reliable system has been reached. In an attempt to study TS, a murine model of the disease has been developed using gene targeting approach (19). As homozygous $T s c 2$ mutations are embryonic lethal, $T s c 2^{+/-}$mice were used in this case. $T s c 2^{+/}$animals are characterized by the development of renal, liver, lung and extremity tumors, although their pregnancy survival is normal. Angiosarcomas appearing on the extremities of the mice, one of the types of tumors present in $T s c 2^{+/-}$animals, were seen in $\sim 7 \%$ of cases. Histologically they were classified as invasive sarcomas 
characterized by slow growth and no distant metastases. A relatively low growth rate and no conversion to pathological malignancy resemble the same type of lesions appearing in TS-affected humans. Thus, a cell line (called TSC2ang1) has been established from one of the angiosarcomas of $T s 2^{+/-}$ mice.

As TSC2ang1 is currently the only TS-associated line available in American Tissue Culture Collection and the cells have not been characterized morphologically or functionally, we decided to evaluate their fitness for studies of TS-associated pathologies. Our main goal was to determine the effect of Akt/PKB and Erk activation on cell proliferation, as these two kinases are thought to drive tumor growth in TSassociated pathologies. Using phospho-specific antibodies, detecting active, phosphorylated forms of kinases, we also studied the response of TSC2ang 1 to Akt/PKB and Erk inhibition. In addition, we evaluated the chromosome stability of the line.

\section{Materials and methods}

Cell culture. TSC2ang1, CHO (Chinese hamster ovary) and SW 480 (human colorectal adenocarcinoma) cells (all from ATCC) were cultured in Dulbecco modified Eagle's medium supplemented with $10 \%$ heat-inactivated FBS, 2 mM L-glutamine, $100 \mathrm{U} / \mathrm{ml}$ penicillin and $100 \mu \mathrm{g} / \mathrm{ml}$ streptomycin (all from Invitrogen, Carlsbad, CA) in $5 \% \mathrm{CO}_{2}$ at $37^{\circ} \mathrm{C}$. Cells were passaged by trypsinization whenever they reached confluence, for no more than 15 times.

Cell proliferation assays. TSC2ang1 cells were seeded onto 24-well plates at a density of 100,000 per well in DMEM with $10 \% \mathrm{FCS}$, in triplicate. After $24 \mathrm{~h}$ (day 0 ), when all cultures were in expotential phase of growth, $100 \mathrm{nM}$ wortmannin, $20 \mathrm{nM}$ rapamycin, $10 \mu \mathrm{M}$ U0126 or $50 \mu \mathrm{M}$ PD98059 were added to respective wells. Cells were cultured with replacement of medium and inhibitors every $24 \mathrm{~h}$ to minimize possible degradation of drugs. Two cell lines CHO and SW 480 treated in the same manner served as controls. After $72 \mathrm{~h}$ from the first administration of drugs, medium was discarded and cells were washed with PBS (phosphate-buffered saline), then were stained with $0.1 \%$ crystal violet in $30 \%$ methanol. After 10 min of staining wells were rinsed carefully 3 times with tap water and air-dried. The following day stained cells were lysed with 2\% Triton X-100 (Sigma-Aldrich, St. Louis, MO) and absorbance was read at $540 \mathrm{~nm}$ in an ELISA plate reader (SLT Labinstruments, Salzburg, Austria). The proliferation test was repeated 3 times. Results are presented as a ratio of absorbance of treated samples to the absorbance of untreated control.

Protein isolation and Western blotting. Cells were washed in PBS and homogenized with RIPA lysis buffer (20 mM Tris pH 7.5, $150 \mathrm{mM} \mathrm{NaCl}, 1 \%$ Nonidet P-40, $0.5 \%$ sodium deoxycholate, $1 \mathrm{mM}$ EDTA, $0.1 \%$ SDS) with $50 \mathrm{mM}$ sodium fluoride and $1 \mathrm{mM}$ sodium orthovanadate, supplemented with 1X Complete protease inhibitor (Roche, Indianapolis, IN) and Phosphatase inhibitor cocktail I (Sigma-Aldrich). Lysates were stored at $-80^{\circ} \mathrm{C}$. For Western blotting, $20 \mu \mathrm{g}$ of protein from each sample were subjected to SDS PAGE. Afterwards,

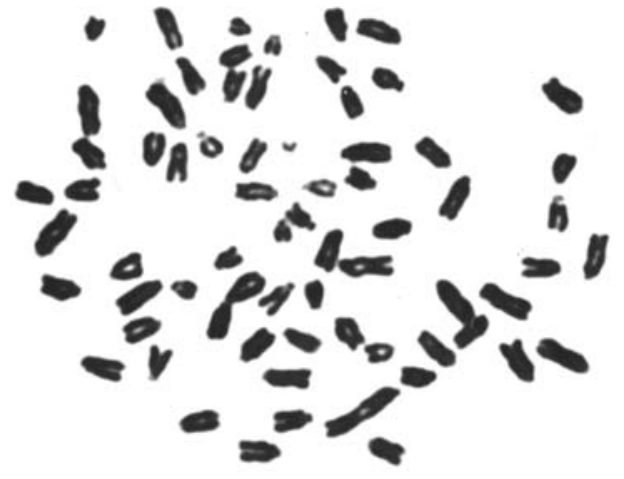

Figure 1. Typical pattern of chromosomes in the TSC2ang1 cell line.

the proteins from gels were transferred onto PVDF membranes. Equal protein loading was verified with Poceau S, then membranes were washed and blocked with $5 \%$ non-fat milk in TBST. Next, the blots were subjected to primary and secondary, HRP-conjugated, antibodies and proteins of interest were visualized by West-Pico chemiluminescence detection kit (Pierce, Rockford, IL).

Antibodies. Antibodies against: phospho-S6K1, phospho4E-BP1, phospho-Erk, phospho-RSK1, phospho-Akt, total Akt, total Erk, and secondary antibodies were purchased from Cell Signaling Technologies (Beverly, MA). Anti-hamartin polyclonal antibody (Santa Cruz Biotechnology, CA) was directed against 300-amino acid $\mathrm{N}$-terminal region of human hamartin and anti-tuberin monoclonal antibody (Novocastra, Newcastle upon Tyne, UK) was directed against 240 amino acid N-terminal region of human tuberin.

Karyotyping. Exponentially growing TSC2ang1 cells were incubated with colcemid $(0.5 \mu \mathrm{g} / 10 \mathrm{ml}$ of medium $)$ at $37^{\circ} \mathrm{C}$ for $2 \mathrm{~h}$. Cells were harvested by trypsinization and treated with $0.075 \mathrm{KCl}$ at $37^{\circ} \mathrm{C}$ for $15 \mathrm{~min}$. After fixation in methanolacetic acid solution the cells were spotted onto slides, air-dried and stained with $10 \%$ Giemsa solution. The chromosome number was calculated in 50 metaphase plates.

\section{Results}

Karyotype. A typical karyotype of TSC2ang1 cells is shown in Fig. 1. The median number of chromosomes was $71 \pm 4$. The arithmetic mean of the chromosome number was 70.73 $(\mathrm{SD} \pm 1.73)$. One Robertsonian translocation (RT), typical for mouse species, was found in $48 \%$ of metaphasal plates, while two RTs were found in $6 \%$ of plates.

U0126 efficiently suppresses Erk activation in TSC2ang1. As we have recently shown (17) Erk activation is associated with at least two types of TS-derived tumors (renal angiomyolipoma, AML, and subependymal giant cell astrocytoma, SEGA). Two commercially available MEK1/2 inhibitors, U0126 and PD98059, were tested in a preliminary experiment (data not shown). At the manufacturer's recommended doses U0126 had a stronger inhibiting effect on Erk activation, hence we studied U0126 in greater detail. While determining the efficiency of U0126 to suppress Erk activation in 


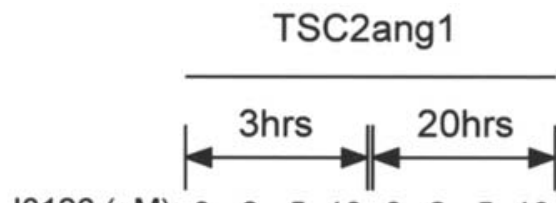

J0126 $(\mu \mathrm{M}): \begin{array}{llllllll}0 & 2 & 5 & 10 & 0 & 2 & 5 & 10\end{array}$

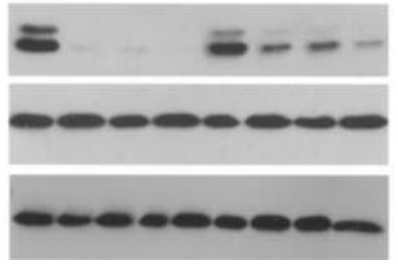

pErk Y204

total Erk

p4E-BP1 S65/T70

Figure 2. Erk inactivation by U0126, a Mek1/2 inhibitor. Cells were incubated for 3 or $20 \mathrm{~h}$ in the presence of different U0126 concentrations. Equal protein loading was confirmed by total-Erk antibody reaction. MEK inhibition results in hypophosphorylation of Erk on tyrosine 204. Lack of effect of Erk inhibition on mTOR pathway is shown by equal 4E-BP1 phosphorylation (at S65/T70).

TSC2ang1 we noted high sensitivity of these cells to this inhibitor administrated even at low concentrations (Fig. 2). After $3 \mathrm{~h}$ of incubation, the concentration as low as $2 \mu \mathrm{M}$ was able to completely abolish Erk activation. On the other hand, when incubation time was extended to $20 \mathrm{~h}$, even the highest evaluated U0126 concentration of $10 \mu \mathrm{M}$ did not completely suppress phosphorylation of Erk. At the same time, we noted that even complete Erk inhibition did not influence phosphorylation of 4E-BP1, one of the mTOR targets, controlling translation initiation of numerous proteins, including those triggering cell cycle progression.

Wortmannin inhibits PI3K more selectively than LY294002. PI3K inhibitors, such as LY294002 and wortmannin, act selectively on the Akt pathway only at a certain concentration, above which they display unspecific, off-target effects (20). Thus, we tested these inhibitors at several concentrations to determine the appropriate dose. Wortmannin was able to suppress effectively phosphorylation of Akt by $\sim 90 \%$ of its control activity, only in its highest concentration (100 nM), the optimal concentration of LY294002 was $25 \mu \mathrm{M}$ (Fig. 3). Higher concentrations of LY294002 $(50 \mu \mathrm{M})$, were not only unselective, but also toxic. We noted, that at this concentration of LY294002, TSC2 protein was undetectable in the lysates due to unknown mechanisms. Since LY294002 at high doses has been also shown to interact directly with mTOR and inhibit its activity $(20,21)$, in order to prevent this phenomenon from influencing our results, we decided to use only wortmannin in further experiments.

mTOR, Akt and Erk inhibitors affect proliferation of TSC2ang1, CHO and SW480. In order to assess whether Akt or Erk is a kinase driving proliferation of cells, we compared the effect of Akt, Erk and mTOR inhibition in TSC2ang1, CHO and SW480 cells. The proliferative potential of the latter two had been shown before to be controlled by Akt or Erk, respectively $(22,23)$. Although U0126 seems to be a better inhibitor of Erk than PD98059, since their mode of action is different, we included both drugs in this study. To find out
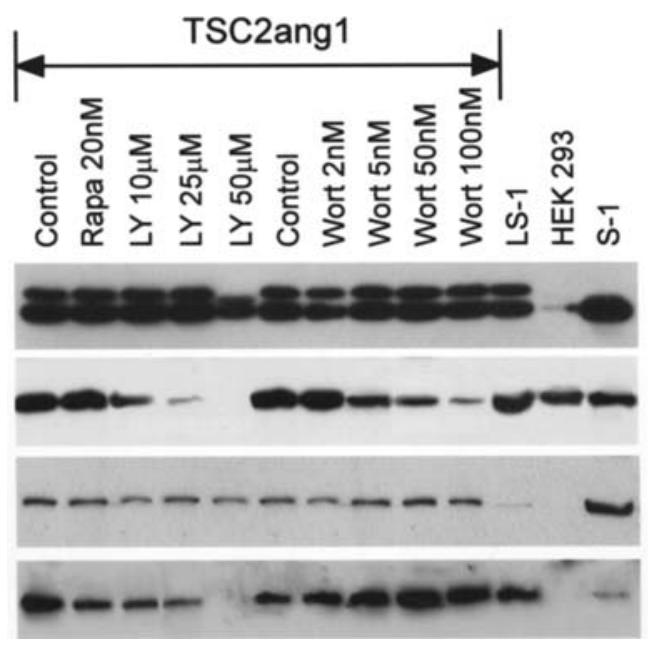

pERK Y204

pAkt S473

TSC1

TSC2

Figure 3. Effects of LY294002 and wortmannin on Akt activation in TSC2ang1 cells. Cells were starved for $24 \mathrm{~h}$ in serum-free DMEM and then incubated for $3 \mathrm{~h}$ with respective inhibitor concentration. Next, 10\% FBS was added and proteins were harvested. Activation of Erk by its phosphorylation on Y204 served as control for the specificity of inhibition. Levels of both proteins forming the tuberous sclerosis complex, TSC1 and TSC2, were also determined. Additional protein lysates were used as control: LS-1, cell line derived from TS-associated human subependymal giant cell astrocytoma; HEK 293, human embryonic kidney cell line; S-1, cell lysate from TS-associated human subependymal giant cell astrocytoma.

whether the inhibition of targeted pathways affects mTOR, we determined this by Western blot phosphorylation of S6K1 kinase (on T389) which had been reported to be phosphorylated by active mTOR (24) (Fig. 4A). In all tested cells U0126 and, to a lesser extent, PD98059 inhibited Erk phosphorylation, with no effect on the activity of mTOR, which was determined by S6K1 phosphorylation on threonine 389. At the same time wortmannin strongly influenced S6K1 phosphorylation on this residue, which can explain its strong anti-proliferative activity in all three cell lines (see Fig. 4B). Erk inhibition in TSC2ang1 upon U0126 and PD98059 administration did not significantly decrease proliferation of TSC2ang1. On the other hand, rapamycin was a very potent inhibitor of growth for these cells. Growth arrest was also observed in two other cell lines treated with rapamycin, but to a lesser extent.

\section{Discussion}

Basic research aiming ultimately at TS treatment is mainly based on animal and cellular studies. Due to ethical and economic reasons, animal (mouse and rat) models should only be used in advanced stages of studies, while cellular systems are needed to screen effectiveness of new drugs. In the current study, we evaluated the fitness of the $\mathrm{TSC}^{+/-}$cell line TSC2ang 1 from mouse sarcoma, the only commercially available TS-related cell line, that could be used for the studies on pathophysiology of TS, as well as for measuring the effect of novel drugs. The first research using this cell line was performed by Govindarajan et al in 2003 (25). The authors observed high levels of active Erk and showed that introduction of a dominant-negative MEK led to decreased tumorigenesis of the line in vivo. In another study, Ma et al 
A
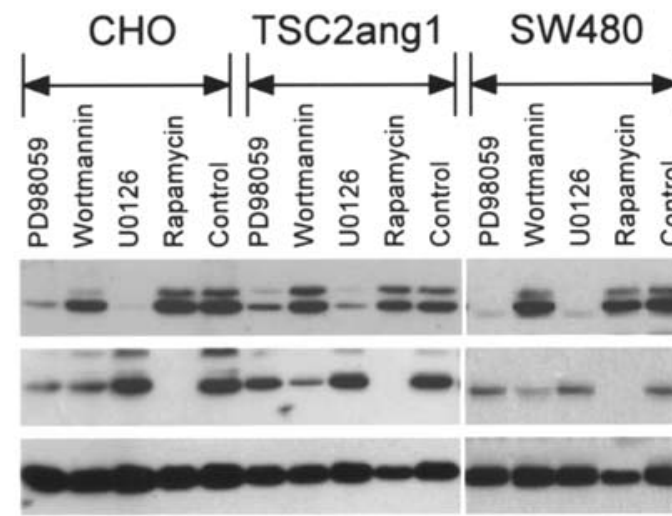

pErk Y204

pS6K1 T389

p4E-BP1 S65/T70

B

$\mathrm{CHO}$

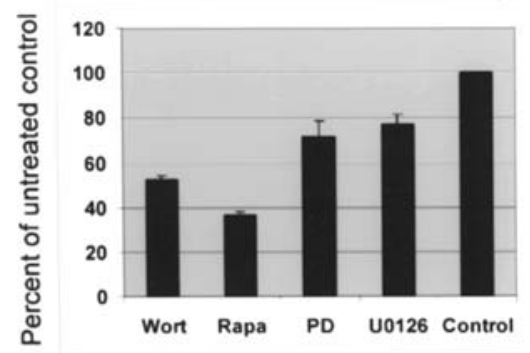

TSC2ang1

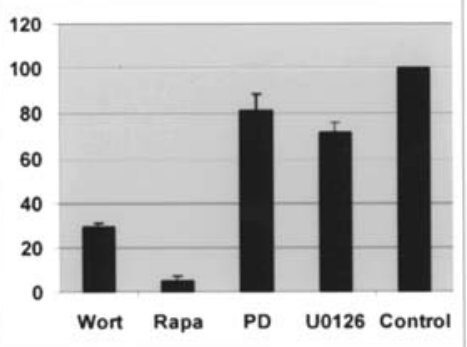

SW480

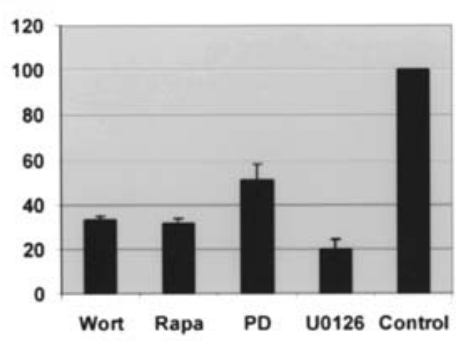

Figure 4. Effect of optimal concentrations of inhibitors of Akt (100 nM wortmannin), Erk (50 $\mu$ M PD98059, $10 \mu \mathrm{M}$ U0126) and mTOR (20 nM rapamycin) was analyzed by Western blot (A) and proliferation assay (B) as described in Materials and methods. Mean values of one of three independent experiments in triplicate are shown. Error bars represent standard error.

(26) evaluated tumorigenic potential of TSC2ang1 line upon transfection with wild-type TSC2, TSC2 $2^{\text {S664A/S540A }}$ (nonphosphorylable by Erk) or TSC2 $2^{\text {S664D/S540D }}$ (posphorylationmimicking mutant). Cells were cultured in soft agar or injected into athymic mice. In both assays only Erk-nonphosphorylable mutant decreased the tomorigenic potential of the line. However, until now, there have been no reports on other pathways engaged in these cells which could determine validity of the cell line as a model of tumors developing in TS.

Tumor development in TS is usually triggered by a secondhit genetic event, most often loss of heterozygosity ( $\mathrm{LOH}$ ) for the affected gene. However, there are TS-associated tumors which remain heterozygous. Brain and some heart lesions are commonly such cases. Although the first hit was evident in the TSC2ang 1 cells, for they were derived from an animal in which one TSC2 locus was replaced with NEO-cassette, the second allele remained intact, as indicated by tuberin (TSC2) expression (Fig. 3). Therefore, a different mechanism must underlie neoplastic transformation of these cells.

In a recent research that we performed on TS-associated SEGAs (17) we determined that proliferation potential of tumor cells is driven by Erk. This kinase has been shown to activate eukaryotic translation initiation factor 4B (eIF4B) in the mTOR-dependent and -independent manner (27).
Cells derived from SEGA were not only Erk-hyperactivated, but also were very resistant to Erk inhibitors, even in high concentrations. Nevertheless, even low degree of Erk inhibition led to marked decrease of proliferation rate. TSC2ang1 line demonstrates different profile of activation. Erk phosphorylation in TSC2ang1 is not as potent as in SEGAs and is easily abolished by both Erk inhibitors, U0126 and PD98059. As expected, the line responds eagerly to rapamycin, a selective mTOR inhibitor. At the same time, two control cell lines, known to be Akt or Erk-dependent, were tested. In both cases rapamycin did not suppress cell growth to such level as it was in the case of TSC2ang1 cells. In all cells $100 \mathrm{nM}$ dose of wortmannin had significant effect on cell proliferation. We speculate, that off-target effect of this inhibitor could play a role in this setting, as it is in the case of limfoblastoid cells (20). Since the targets of wortmannin, PI3K and mTOR are structurally related (28) it is likely, that in high doses this molecule inhibits both kinases, similarly to LY294002. This could explain why Erk-driven SW480 cells were partially inhibited by wortmannin.

Although preliminary evaluation of two MEK/Erk inhibitors pointed to U0126 as the more effective molecule, in the proliferation test we employed both drugs. Their mode of action is different (PD98059 prevents activation of MEK, while U0126 inhibits activated form of this kinase) therefore 
parallel usage of two such molecules additionally validates our results. As the proliferation of ki-Ras-driven SW480 cells dropped to $20 \%$ when U0126 was administered in the recommended dose of $10 \mu \mathrm{M}, 50 \mu \mathrm{M}$ PD98059 decreased this ratio to $\sim 50 \%$. At the same time, these drugs had only a very modest effect on the growth of $\mathrm{CHO}$ and TSC2ang1 (both $\sim 75 \%$ of control). These results argue, that TSC2ang1 cells, although engaging the MEK/Erk pathway, do not depend on it as much as on mTOR. The latter one, when inhibited with rapamycin, completely abolished their proliferation. Although this was expected, since rapamycin was effectively used in TS in the first clinical trials to reduce the size of tumors (29), this result proves the validity of this line as the model of mTOR hyperactivation, such as one found in skin lesions developing in TS patients.

Although there are differences in the drug response pattern of TSC2ang 1 and primary cells derived from TSrelated brain tumors, they can be explained by at least two facts. Firstly, SEGAs and skin lesions demonstrate different proliferation potential. SEGA growth may disturb the flow of the cerebrospinal fluid, which is a common cause of their excision, and may even metastasize (30), skin lesions in TS are stable and their cells do not form tumors. Secondly, inactivating TSC1 and TSC2 mutations appear in most other types of TS-associated lesions, but not in the brain or heart $(10,13)$. Thus, we think that in SEGAs haploinsufficiency resulting from mutation of only one allele of TSC1 or TSC2 may be supplemented by Erk activation, and that only this combination of events leads to tumor progression. On the other hand, according to our recent experiments performed on TS-associated skin lesion samples (unpublished data), this type of lesions may not carry Erk hyperactivation and thus, may behave less aggressively. From this point of view, it seems that TSC2ang1 may be a good model for studying skin lesions found in TS.

Autonomous growth of the TSC2ang1 cell line is evidenced by its polyploidisation. As previously reported $(31,32)$, rodent cell lines with a diploid or nearly diploid chromosome number do not have the ability of autonomous growth, and require e.g. growing on feeder layers. Karyotyping of TSC2ang1 shows that the model number of chromosomes is 71 . We did not find differences in the number of chromosomes in early and late passages (data not shown), thus, it seems that the rate of polyploidy, and thus, the cell line, is stable. Therefore, we conclude, that TSC2ang1 cells can be a useful model in TS-related research especially for those pathologies, where loss of heterozygosity for TSC2 is not found.

\section{Acknowledgements}

This work was supported by Polish Ministry of Science and Higher Education grant \# N406 031/0916, by The Children's Memorial Health Institute grant \# 107/05 and by The Medical University of Warsaw grant \# 1M15/W2/07.

\section{References}

1. van Slegtenhorst M, de Hoogt R, Hermans C, et al: Identification of the tuberous sclerosis gene TSC1 on chromosome 9q34. Science 277: 805-808, 1997.

2. European Tuberous Sclerosis Consortium: Identification and characterization of the tuberous sclerosis gene on chromosome 16. Cell 75: 1305-1315, 1993.
3. Inoki $\mathrm{K}$, Li Y, Zhu T, Wu J and Guan KL: TSC2 is phosphorylated and inhibited by Akt and suppresses mTOR signalling. Nat Cell Biol 4: 648-657, 2002.

4. Roux PP, Ballif BA, Anjum R, Gygi SP and Blenis J: Tumorpromoting phorbol esters and activated Ras inactivate the tuberous sclerosis tumor suppressor complex via p90 ribosomal S6 kinase. Proc Natl Acad Sci USA 101: 13489-13494, 2004.

5. Jozwiak J, Jozwiak S, Grzela T and Lazarczyk M: Positive and negative regulation of TSC2 activity and its effects on downstream effectors of the mTOR pathway. Neuromolecular Med 7: 287-296, 2005.

6. Sonenberg $\mathrm{N}$ and Pause A: Signal transduction. Protein synthesis and oncogenesis meet again. Science 314: 428-429, 2006.

7. Kimball SR, Shantz LM, Horetsky RL and Jefferson LS: Leucine regulates translation of specific mRNAs in L6 myoblasts through mTOR-mediated changes in availability of eIF4E and phosphorylation of ribosomal protein S6. J Biol Chem 274: 11647-11652, 1999

8. Takuwa N, Fukui Y and Takuwa Y: Cyclin D1 expression mediated by phosphatidylinositol 3-kinase through mTORp70(S6K)-independent signaling in growth factor-stimulated NIH 3T3 fibroblasts. Mol Cell Biol 19: 1346-1358, 1999.

9. Mamane Y, Petroulakis E, LeBacquer O and Sonenberg N: mTOR, translation initiation and cancer. Oncogene 25: 6416-6422, 2006.

10. Henske EP, Wessner LL, Golden J, et al: Loss of tuberin in both subependymal giant cell astrocytomas and angiomyolipomas supports a two-hit model for the pathogenesis of tuberous sclerosis tumors. Am J Pathol 151: 1639-1647, 1997.

11. Jozwiak S, Dabora S, Kasprzyk-Obara J, Domanska-Pakiela D and Grajkowska W: Tests for loss of heterozygosity in tuberous sclerosis. Przegl Lek 58 (Suppl 1): 12-15, 2001.

12. Knowles MA, Habuchi T, Kennedy W and Cuthbert-Heavens D: Mutation spectrum of the 9q34 tuberous sclerosis gene TSC1 in transitional cell carcinoma of the bladder. Cancer Res 63: 7652-7656, 2003.

13. Meikle L, McMullen JR, Sherwood MC, et al: A mouse model of cardiac rhabdomyoma generated by loss of Tsc 1 in ventricular myocytes. Hum Mol Genet 14: 429-435, 2005.

14. Jansen FE, Notenboom RG, Nellist M, et al: Differential localization of hamartin and tuberin and increased S6 phosphorylation in a tuber. Neurology 63: 1293-1295, 2004.

15. Mizuguchi M, Ikeda K and Takashima S: Simultaneous loss of hamartin and tuberin from the cerebrum, kidney and heart with tuberous sclerosis. Acta Neuropathol 99: 503-510, 2000.

16. Tabuchi S, Takigawa H, Oka A, Mizuguchi M, Horie Y and Watanabe T: Subependymal giant cell astrocytoma with positive tuberin expression - case report. Neurol Med Chir 43: 616-618, 2003.

17. Jozwiak J, Grajkowska W, Kotulska K, et al: Brain tumor formation in tuberous sclerosis depends on Erk activation. Neuromolecular Med (In press).

18. Gera JF, Mellinghoff IK, Shi Y, et al: AKT activity determines sensitivity to mammalian target of rapamycin (mTOR) inhibitors by regulating cyclin D1 and c-myc expression. J Biol Chem 279: 2737-2746, 2004.

19. Onda H, Lueck A, Marks PW, Warren HB and Kwiatkowski DJ: Tsc2(+/-) mice develop tumors in multiple sites that express gelsolin and are influenced by genetic background. J Clin Invest 104: 687-695, 1999.

20. Wlodarski P, Kasprzycka M, Liu X, et al: Activation of mammalian target of rapamycin in transformed B lymphocytes is nutrient dependent but independent of Akt, mitogen-activated protein kinase/extracellular signal-regulated kinase kinase, insulin growth factor-I, and serum. Cancer Res 65: 7800-7808, 2005.

21. Sabatini DM, Erdjument-Bromage H, Lui M, Tempst P and Snyder SH: RAFT1: a mammalian protein that binds to FKBP12 in a rapamycin-dependent fashion and is homologous to yeast TORs. Cell 78: 35-43, 1994.

22. Kitamura T, Ogawa W, Sakaue H, et al: Requirement for activation of the serine-threonine kinase Akt (protein kinase B) in insulin stimulation of protein synthesis but not of glucose transport. Mol Cell Biol 18: 3708-3717, 1998.

23. McCoy MS, Bargmann CI and Weinberg RA: Human colon carcinoma Ki-ras2 oncogene and its corresponding protooncogene. Mol Cell Biol 4: 1577-1582, 1984.

24. Burnett PE, Barrow RK, Cohen NA, Snyder SH and Sabatini DM: RAFT1 phosphorylation of the translational regulators p70 S6 kinase and 4E-BP1. Proc Natl Acad Sci USA 95: 1432-1437, 1998. 
25. Govindarajan B, Mizesko MC, Miller MS, et al: Tuberous sclerosis-associated neoplasms express activated p42/44 mitogen-activated protein (MAP) kinase, and inhibition of MAP kinase signaling results in decreased in vivo tumor growth. Clin Cancer Res 9: 3469-3475, 2003.

26. Ma L, Chen Z, Erdjument-Bromage H, Tempst P and Pandolfi PP: Phosphorylation and functional inactivation of TSC2 by Erk implications for tuberous sclerosis and cancer pathogenesis. Cell 121: 179-193, 2005

27. Shahbazian D, Roux PP, Mieulet V, et al: The mTOR/PI3K and MAPK pathways converge on eIF4B to control its phosphorylation and activity. EMBO J 25: 2781-2791, 2006.

28. Barylko B, Wlodarski P, Binns DD, et al: Analysis of the catalytic domain of phosphatidylinositol 4-kinase type II. J Biol Chem 277: 44366-44375, 2002.
29. Franz DN, Leonard J, Tudor C, et al: Rapamycin causes regression of astrocytomas in tuberous sclerosis complex. Ann Neurol 59: 490-498, 2006.

30. Telfeian AE, Judkins A, Younkin D, Pollock AN and Crino P: Subependymal giant cell astrocytoma with cranial and spinal metastases in a patient with tuberous sclerosis. Case report. J Neurosurg 100: 498-500, 2004.

31. Castro-Munozledo F: Development of a spontaneous permanent cell line of rabbit corneal epithelial cells that undergoes sequential stages of differentiation in cell culture. J Cell Sci 107: 2343-2351, 1994.

32. Negrel R, Rampal P, Nano JL, Cavenel C and Ailhaud G: Establishment and characterization of an epithelial intestinal cell line from rat fetus. Exp Cell Res 143: 427-437, 1983. 